\title{
Quantized (or not quantized) thermal Hall effect and oscillations in the thermal conductivity in the Kitaev spin liquid candidate $\alpha-\mathrm{RuCl}_{3}$.
}

1. Oscillations of the thermal conductivity observed in the spin-liquid state of $\alpha-\mathrm{RuCl}_{3}$

Authors: Peter Czajka, Tong Gao, Max Hirschberger, Paula Lampen-Kelley, Arnab

Banerjee, Jiaqiang Yan, David G. Mandrus, Stephen E. Nagler, N. P. Ong

Nature Physics 17, 915-919 (2021)

2. The planar thermal Hall conductivity in $\alpha-R u C l_{3}$

Authors: Peter Czajka, Tong Gao , Max Hirschberger , Paula Lampen-Kelley ,

Arnab Banerjee4, Nicholas Quirk, David G. Mandrus, Stephen E. Nagler, and N.

P. Ong

https://arxiv.org/abs/2111.0xxxx

3. Robustness of the thermal Hall effect close to half-quantization in a field-induced spin liquid state

Authors: J. A. N. Bruin, R. R. Claus, Y. Matsumoto, N. Kurita, H. Tanaka, H.

Takagi

https://arxiv.org/abs/2104.12184

4. Evidence of a Phonon Hall Effect in the Kitaev Spin Liquid Candidate $\alpha-\mathrm{RuCl}_{3}$

Authors: É. Lefrançois, G. Grissonnanche, J. Baglo, P. Lampen-Kelley, J. Yan, C. Balz, D. Mandrus, S. E. Nagler, S. Kim, Young-June Kim, N. Doiron-Leyraud, and L. Taillefer

https://arxiv.org/abs/2111.05493

5. Sign Structure of Thermal Hall Conductivity and Topological Magnons for In-Plane Field Polarized Kitaev Magnets

Authors: Li Ern Chern , Emily Z. Zhang, and Yong Baek Kim

Physical Review Letters 126, 147201 (2021)

6. Pseudoscalar $\mathrm{U}(1)$ spin liquids in $\alpha-R u C l_{3}$

Authors: Inti Sodemann Villadiego

http://arxiv.org/abs/2106.05290

Recommended with a Commentary by Patrick A. Lee, MIT 
In 2006 Alexei Kitaev [1] introduced an exactly soluble model which exhibits all the expected features of a quantum spin liquid. This remarkable breakthrough is based on quantum spins on a two dimensional honeycomb lattice with a special highly directional interaction. While the model initially looked unrealistic, the work by Jackeli and Khaliulin [2] showed that the "Kitaev coupling" can be realized in real materials with strong spin-orbit coupling in certain structures. Since that time $\alpha-R u C l_{3}$, a layered compound where $\mathrm{Ru}$ ions sit on a honeycomb lattice has emerged as one of the most promising candidates for the Kitaev model. The importance of other terms in the Hamiltonian is still subject to debate, and the system has an anti-ferromagnetic zig-zag ordering at zero applied magnetic field. However, it is known that upon applying a magnetic field of about 7T parallel to the plane, the antiferromagnetic order is destroyed. There has been growing evidence, though not definitive, that beyond this field a spin liquid state emerges which is eventually replaced by a conventional partially spin polarized state above 11T. A great deal of excitement was injected into the field with the report by Kasahara et al [3] in 2018 that the thermal Hall effect per layer is quantized at $\kappa_{x y} / T=\pi^{2} k_{B}^{2} / 6 h$, which is half of the value expected for a fermion edge state. (Thermal Hall effect refers to the appearance of a thermal gradient perpendicular to a heat current in the presence of a magnetic field). This has been considered to be strong evidence for the existence of an Majorana edge mode. The data from a subsequently paper from the same group (Yokoi et al. [4]) are shown in fig. 1,
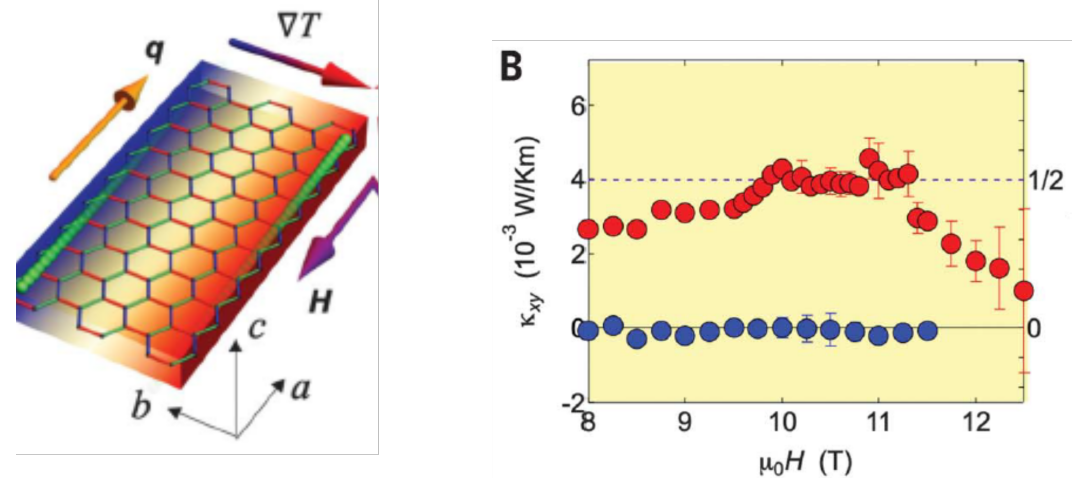

Figure 1: Left panel, geometry of the thermal Hall measurement. Note that the heat current, denoted by q, is parallel to the magnetic field $\mathrm{H}$. Right panel, $\kappa_{x y}$ as a function of magnetic field at $4.8 \mathrm{~K}$. The red and blue data points are for $\mathrm{H}$ along $a$ and $b$ respectively. The dotted line marks the half-quantized value. From Yokoi et al, [4]

There has been a series of rapid recent developments, resulting in a rather large set of recommended papers in this commentary. The short summary is that considerable doubts have been raised on whether the thermal Hall conductivity indeed exhibits the claimed quantization. On the other hand, oscillations have been reported in the thermal conductivity as a function of magnetic field. This is highly unusual for an insulator, and is reminiscent of quantum oscillations in metals. These data raise new questions which show that we are far from understanding the nature of the "spin liquid" like phase that has been under intensive studies in this material.

Before launching into details let me point out some key feature of the observed effect. 
While in the first paper [3] a magnetic field was applied both perpendicular and parallel to the plane, the follow up paper by Yokoi et al [4] showed that the effect is present even when the perpendicular magnetic field is zero. To be specific, the thermal Hall signal arises when a magnetic field is applied along the $a$ direction (defined as perpendicular to the $\mathrm{Ru}-\mathrm{Ru}$ bond, as shown in fig 1 when the field exceeds about 5T. On the other hand, when the field is applied along the $b$ direction (along the Ru-Ru bond) the effect is zero. The latter is easily understood because a Hall current is odd and therefore forbidden under a symmetry of the crystal structure (rotation about the $b$ axis) which is preserved by a magnetic field along $b$. In contrast, for magnetic field along $a$ this symmetry does not exist and the Hall effect is allowed. In this case both the applied magnetic field and the heat current are along the $a$ direction and the effect should be called a planar Hall effect. Unfortunately the term "planar Hall effect" has been usurped by a different phenomenon involving ferromagntic materials: when an external field is applied at an angle to the magnetization, the anisotropy of the resistivity with respect to the magnetization direction generates a transverse voltage which does not change sign when the direction of $B$ is reversed. This effect should really not be referred to as a Hall effect which should be odd in the applied magnetic field, but we are stuck with this misnomer. Nevertheless, in the rest of this comment, I shall refer to what is observed in $\alpha-\mathrm{RuCl}_{3}$ as planar thermal Hall effect. The planar thermal Hall effect is on its own an unusual phenomenon because it suggests that its origin does not arise from a Lorentz force. There is no disagreement that the planar Hall effect exists in $\alpha-\mathrm{RuCl}_{3}$. The arguement is whether it is quantized or not.

A quantized $\kappa_{x y} / T$ should show a plateau pinned to the quantized value as a function of magnetic field and temperature. In [4] the thermal Hall signal shows a plateau at the half quantized value over a relatively small range in magnetic field (between 10 to $11 \mathrm{~T}$ along b) and in temperature (between 3.5 to $6 \mathrm{~K}$ ), as shown fig 1 . It is worth noting that in the earlier paper by Kasahara et al. [3] the parallel magnetic field where the quantization was observed was in the range 6.5 to $8.7 \mathrm{~T}$ (depending on the perpendicular component), and this field range coincides with where spin liquid phase is expected. The new range of 10 to 11T [4] no longer has a clear association with the spin liquid regime. The recommended paper 1 by Czajka et al. is mainly concerned with the oscillations in $\kappa_{x x}$, a subject to which we will return later, but they made the point that they did not reproduce the quantized $\kappa_{x y} / T$. Instead they found that $\kappa_{x y}$ evolves smoothly with temperature with no sign of a plateau. Furthermore the magnitude of their signal at $5 \mathrm{~K}$ and $10 \mathrm{~T}$ is about 60 percent less than the half-quantized value reported by [4]. Bruin et al. (recommended paper 3) reported measurements over a large magnetic field and temperature range. Some of their data are reproduced in fig. 2. The data show a gradual evolution of the magnetic field dependence as the temperature is varied. At a fixed temperature there is no clear plateau at the halfquantized value, except perhaps at $6.5 \mathrm{~K}$. Nevertheless, Bruin et al. came to the conclusion that their data support half quantization for magnetic field greater than $10 \mathrm{~T}$ and over a temperature range below $6.5 \mathrm{~K}$. The right panel in fig. 2 shows their data plotted vs $\mathrm{T}$ for a set of magnetic fields. It seems that a plateau may be visible at $10.3 \mathrm{~T}$, but the value is 20 percent below the half-quantized value. At fields above $12 \mathrm{~T}$ the error bars are getting progressively larger and it may be risky to draw any firm conclusion. It should be noted that Bruin et al do not support quantization in the range between $7 \mathrm{~T}$ and $10 \mathrm{~T}$ where the spin liquid phase is expected to reside. 

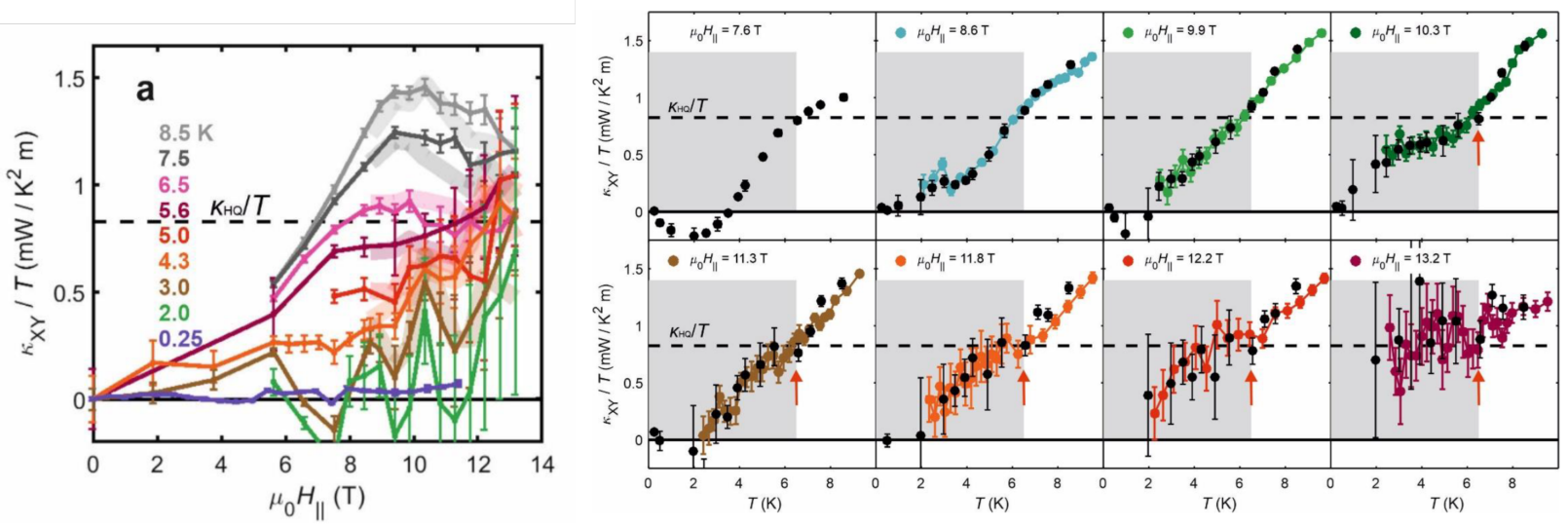

Figure 2: Left panel. $\kappa_{x y} / T$ vs magnetic field $\mathrm{H}$ for a set of temperatures. Right panel. $\kappa_{x y} / T$ vs $T$ for a set of parallel magnetic field. Black data points are field sweeps at fixed $\mathrm{T}$ and color points are temperature sweeps at fixed field. From Bruin et al, recommended paper 3

In a second paper, Czajka et al (recommended paper 2) extended their measurements to a broader temperature and magnetic field range, as shown in fig. 3. Their data show a smooth evolution with $\mathrm{T}$ and $\mathrm{H}$ and no sign of plateau behavior. Furthermore, they emphasize that the data go rapidly to zero at low temperature, in strong contrast to a constant $\kappa_{x y} / T$ that is expected for fermionic edge modes. The temperature dependence of their data is more reminiscent of Bose-Einstein distribution.

Indeed, theoretical work by Matsumoto and Murakami [5] showed that a bosonic mode such as magnon can show a thermal Hall effect if there are Berry curvatures associated with the magnon band. Such Berry curvatures are typically present and can give rise to thermal Hall conductivity if enough symmetry such as inversion and time reversal are broken. The physical interpretation is that the heat is being carried by edge currents due to the anomalous velocity which is given by the cross product of the Berry curvature and the gradient of the confining potential at the edge. In recommended paper 5, Chern et al. worked out the magnon dispersion in a realistic model for $\alpha-R u C l_{3}$ when the system is partially spin polarized by the magnetic field, in the absence of any anti-ferromagnetic ordering. This model should be applicable in the regime of high magnetic field, beyond the purported spin liquid regime. They found that the lowest mode has a Chern number of unity. Czajka et al fit their temperature data using the formula from Matsumoto and Murakami, assuming a flat bosonic band and found a good fit for a Chern number of unity. Furthermore, their fit yielded a mode frequency which matches well the frequency of a sharp mode observed by electron spin resonance (ESR) over a broad field range that include the spin liquid regime. [6] The picture that emerges is that a magnon-like excitation exists over a broad magnetic field range when the system is polarized and is responsible for the planar Hall effect. This mode is observed by neutron scattering for large field, but not in the "spin liquid" regime in the field range between 7T to 9T. [7] Here a continuum of excitations emerges which may obscure the modes which are observed by ESR. This picture leaves the nature of the "spin liquid" phase unresolved, because from this point of view, the $\kappa_{x y}$ sheds little light on the 

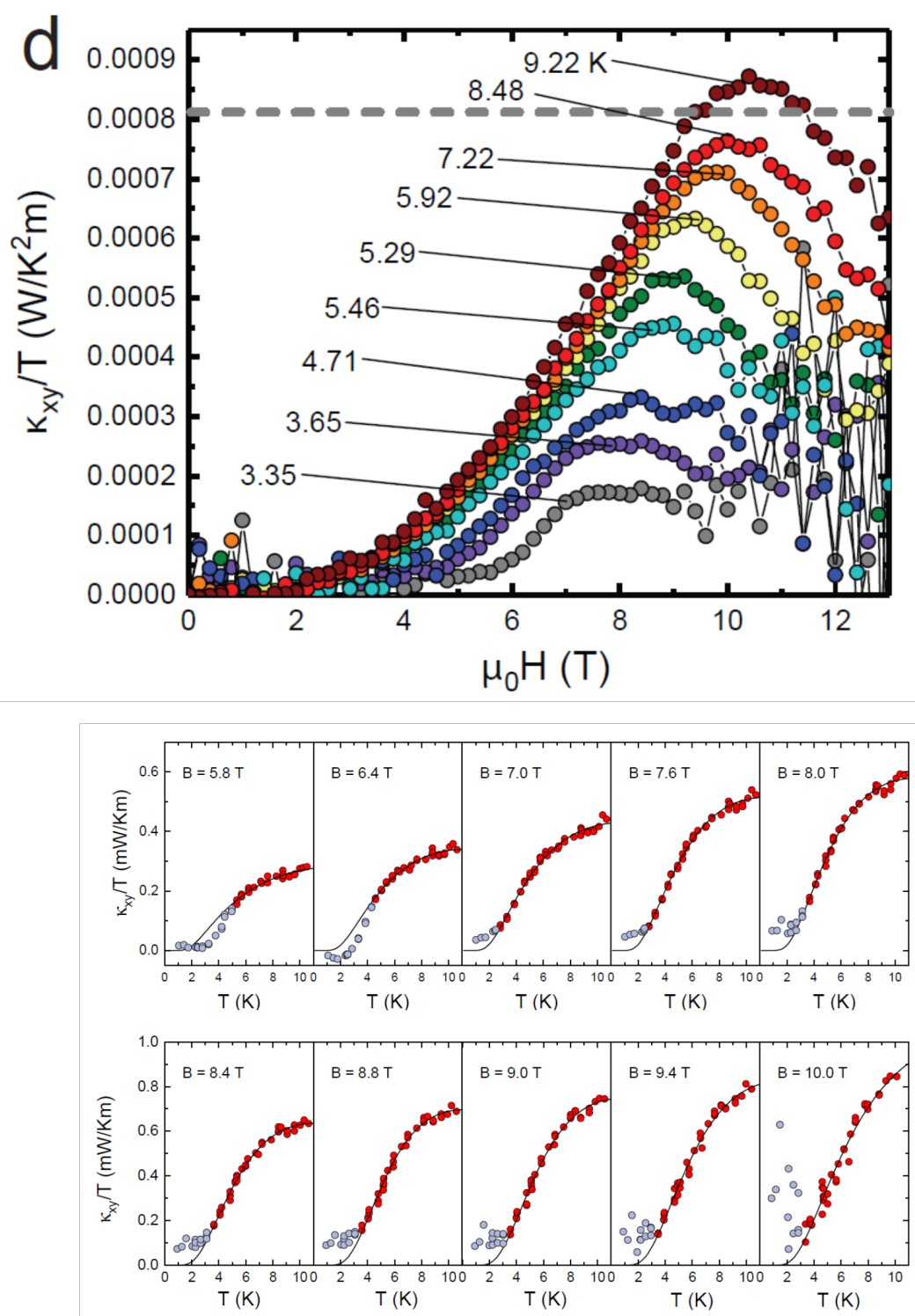

Figure 3: Top panel, $\kappa_{x y} / T$ vs $\mathrm{H}$ for a range of temperatures. Dashed line is the halfquantized value. Bottom panels: $\kappa_{x y} / T$ vs $\mathrm{T}$ for a range of $\mathrm{H}$. Solid lines are the fit to thermal excitations of a bosonic mode whose band has a Chern number of unity, using a formula by Matsumoto and Murakami [5], From Czajka et al. recommended paper 2

nature of the intermediate phase.

For completeness I want to mention the paper by Lefrançois et al (recommended paper 4). They consider a magnetic field either perpendicular or at 45 degrees from the plane and found a thermal Hall effect. Since their signal scales with the thermal conductance $\kappa_{x x}$, they concluded that the heat is being carried by phonons. This is a very interesting observation in itself and adds $\alpha-R u C l_{3}$ to a growing list of materials where phonon thermal Hall effect have been reported. There is little theoretical understanding at this point and the topic is certainly worthy of further investigations. On the other hand, the effect they report is 
expected to vanish once the perpendicular field is zero. Since they did not show any data with zero perpendicular field, their data have no direct bearing on the planar thermal Hall effect under discussion.

We next turn to the report of oscillations in the thermal conductivity reported in recommended paper 1 by Czajka et al. They report that the thermal conductivity exhibits oscillatory structures between $4 \mathrm{~T}$ and $11.5 \mathrm{~T}$ parallel magnetic field. The oscillations onset below $4 \mathrm{~K}$, and the amplitude of the oscillations in $\kappa_{x x} / T$ relative to the background increases with decreasing temperatures, saturating at about $0.5 \mathrm{~K}$. The oscillations appear to be periodic in $1 / \mathrm{H}$ but the period changes at about $7 \mathrm{~T}$. Above $7 \mathrm{~T}$ the period is the same when the field is in the a or b direction, but below $7 \mathrm{~T}$ the period is different. The authors argue that the oscillations are characteristic of the spin liquid and that there is a co-existence phase extending into the ordered phase below $7 \mathrm{~T}$. The periodicity in $1 / \mathrm{H}$ is reminiscent of quantum oscillations in metals, with two important difference. First this is an insulator and second, the field is parallel to the plane.

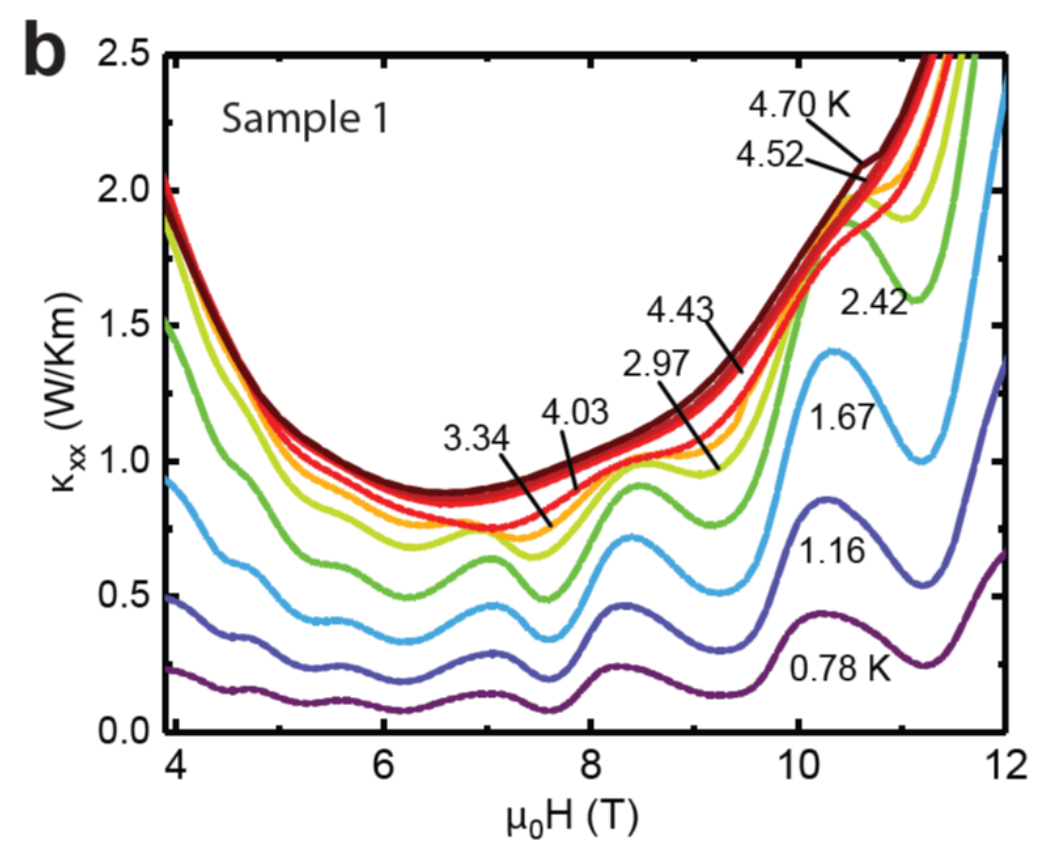

Figure 4: Oscillations the thermal conductivity $\kappa_{x x}$ reported by Czajka et al., recommended paper 2 .

Quantum oscillations have been predicted to occur in a spin liquid with a spinon Fermi surface. [8] The idea is that the external magnetic field generates an emergent gauge magnetic field that is seen by the spinon. Since the spinon carries heat current, quantum oscillations in the thermal conductivity in an insulating spin liquid is possible. It should be noted that the notion of spinon Fermi surface goes against the idea of a gapped spin liquid which supports Majorana edge modes and quantized $\kappa_{x y} / T$, but can be consistent with bosonic mode interpretation discussed earlier. In the original discussion the external magnetic field was assumed to be perpendicular to the plane. [8] In the $\alpha-R u C l_{3}$ structure there exists a symmetry when the field is applied along the $b$ direction which forbids the generation of the 
gauge magnetic field which is odd under this symmetry. However, as discussed earlier, no such symmetry exists when the field is along $a$. Thus the observation of quantum oscillations is consistent with the spinon/gauge field picture when the field is along $a$, but not when the field is along $b$. This puzzle is addressed in the recommended paper 6 by Inti Sodemann Villadiego. He pointed out that while the symmetry strictly forbids a finite $\kappa_{x y}$, in principle oscillations in $\kappa_{x x}$ are allowed as a physical observable. So the problem may have to do with the particular formulation of the $\mathrm{U}(1)$ gauge theory where the gauge magnetic field is a pseudo-scalar, just like a physical magnetic field. He introduced a new formulation where the gauge magnetic field is a scalar. In this formalism quantum oscillations are allowed even with a magnetic field along $b$, but the thermal Hall effect remains zero. He further showed that the new formulation corresponds to the Gutzwiller projection of a mean-field ansatz that is different from the traditional one, and represents a different state under Wen's projective symmetry group classification.
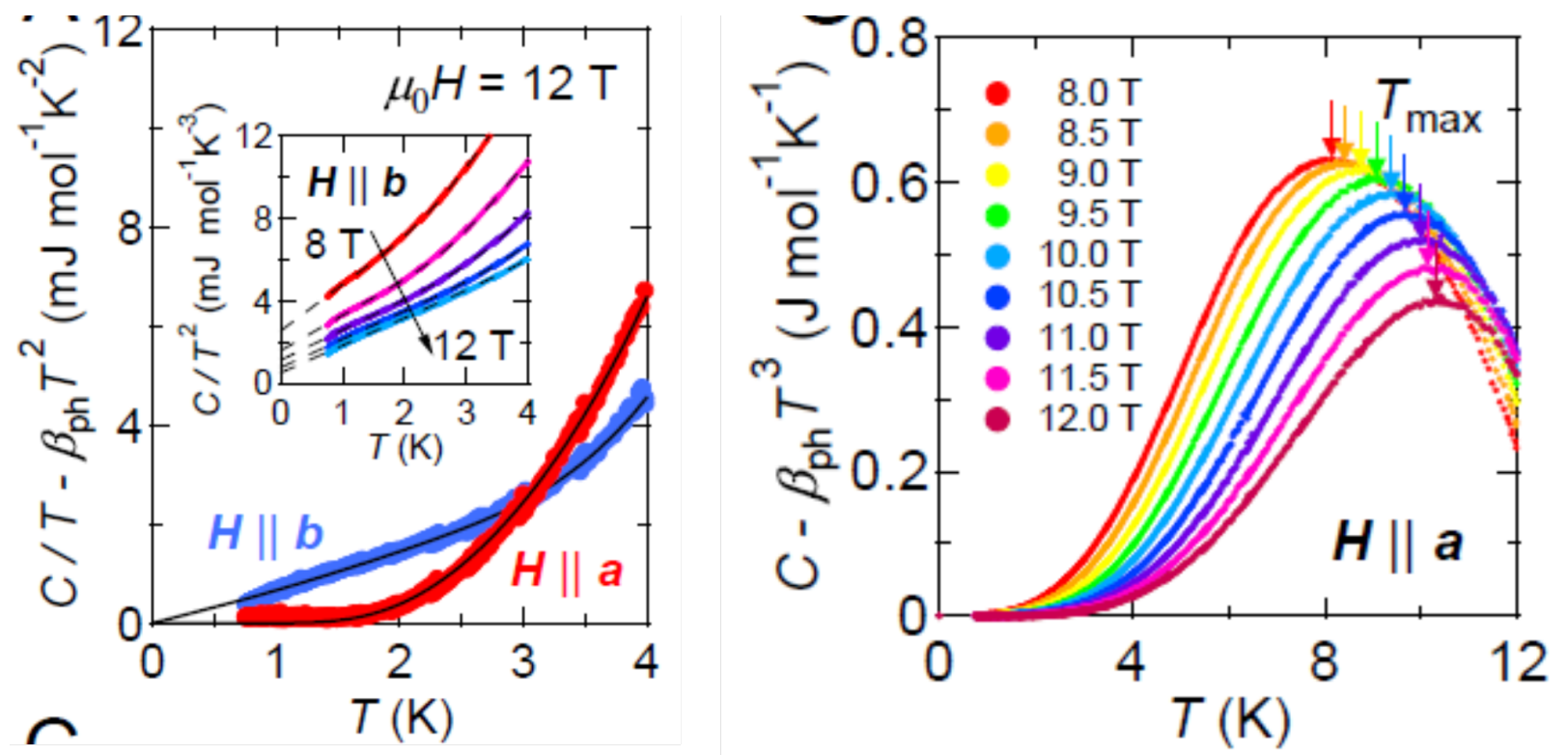

Figure 5: Left panel. Specific heat data after subtracting the phonon contribution vs $\mathrm{T}$ at $12 \mathrm{~T}$ showing contrasting behavior for field along $a$ and $b$. Right panel. Data for field along $a$ for a range of magnetic fields. Note the exponentially small signal around $1 \mathrm{~K}$ over the range of magnetic field where oscillations in the thermal conductivity $\kappa_{x x}$ are reported by Czajka et al., recommended paper 2. Data reproduced from [10]

The observation of quantum oscillations in a spin liquid, if confirmed, represents a significant advance because it can be considered the smoking-gun demonstration of a spinon Fermi surface and the first direct evidence for an emergent gauge magnetic field. While Sodemann's work has removed a major obstacle to this interpretation, a number of open issues remain to be addressed. One issue is that specific heat data has long shown a clear gap at low temperatures for this magnetic field range. [9] More recent data show that the gap corresponds to about $5 \mathrm{~K}$ when the field is along $a$, but could be a power-law behavior when the field is along $b$. [10] According to these data, reproduced in fig. 5, at $1 \mathrm{~K}$ when the oscillations are seen with field along $a$, the spin contribution of the specific heat (after 
subtracting the phonon background) is close to being zero. The specific heat appear to be at odds with the existence of spinon Fermi surfaces and the thermal conductivity should mainly come from phonons. Are the oscillations coming from the phonon mean free path being modulated in some way? This is indeed the interpretation suggested in recommended paper 3. Bruin et al report features in the thermal conductivity as a function of magnetic field which are quite similar to those reported in paper 1 for $\mathrm{H}$ greater than $6 \mathrm{~T}$, but unlike paper 1, they do not see the oscillations between 4 and $6 \mathrm{~T}$. They interpret the dips in the thermal conductivity as originating from the phonons being scattered by soft modes in the magnetic systems, reflecting changes in the magnetic structure. They identify the dips at $6.05 \mathrm{~T}$ and $7.1 \mathrm{~T}$ with transitions in and out of a known intermediate ordered phase and suggest that dips in higher fields are due to some unknown transitions. At the moment it looks like a consensus has not yet been reached on the interpretations of these features in the thermal conductivity.

Finally, at a recent workshop on this subject, Hidenori Takagi (private communication) called attention to the fact that certain aspects of the data shown differences depending on two different growth methods. The Bridgman samples were used by the Kyoto group $[3,4]$ and the Stuttgart group ( Bruin et al., recommended paper 3) while chemical vapor transport (CVT) samples were used by the Princeton group of Czajka et al. Takagi recently acquired CVT samples and reproduced all the structures in $\kappa_{x x}$ shown in fig. 4 . It seems that the modulations are much weaker in the Bridgman samples as reported by Bruin et al. On the other hand, the Bridgman samples typically has $\kappa_{x x}$ that is a factor two larger. Sorting out these sample dependent features will be a necessary next step to make further progress.

\section{References}

[1] Kitaev, A. Anyons in an exactly solved model and beyond. Ann. Phys. 321, 2-111 (2006).

[2] Jackeli, G. Khaliullin, G. Mott Insulators in the Strong Spin-Orbit Coupling Limit: From Heisenberg to a Quantum Compass and Kitaev Models. Phys. Rev. Lett. 102, 017205 (2009).

[3] Kasahara, Y. et al. Majorana quantization and half-integer thermal quantum Hall effect in a Kitaev spin liquid. Nature 559, 227-231 (2018).

[4] Yokoi, T., S. Ma, Y. Kasahara, S. Kasahara, T. Shibauchi, N. Kurita, H. Tanaka et al. "Half-integer quantized anomalous thermal Hall effect in the Kitaev material candidate $\alpha-\mathrm{RuCl}_{3} . "$ Science 373, no. 6554 (2021): 568-572.

[5] Kasahara, Y. et al. Rotational motion of magnons and the thermal Hall effect. Nature 559, 227-231 (2018).

[6] A. N. Ponomaryov et al., Nature of Magnetic Excitations in the High-Field Phase of $\alpha-\mathrm{RuCl}_{3}$, Physical Review Letters, 125, 037202 (2020). 
[7] C. Balz et al., Finite field regime for a quantum spin liquid in $\alpha-R u C l_{3}$, Physical Review B 100, 060405(R) (2019).

[8] Motrunich, O. I. Orbital magnetic field effects in spin liquid with spinon Fermi sea: possible application to kappa-(ET)2Cu2(CN)3. Phys. Rev. B 73, 155115 (2006).

[9] A. Wolter et al,Field-induced quantum criticality in the Kitaev system $\alpha-\mathrm{RuCl}_{3}$. Phys. Rev. B 96, 0144105(R) (2017). (2006).

[10] O. Tanaka et al. Thermodynamic evidence for field-angle dependent Majorana gap in a Kitaev spin liquid. arXiv: 2007.06757 (2020). 